\title{
Unified Impact Theory for Velocity-Changing Effects and Speed Dependencies in Neutral Species Lineshapes
}

\author{
Roger M. Herman \\ Department of Physics, The Pennsylvania State University, 104 Davey Laboratory, University Park, PA 16802, USA \\ Correspondence should be addressed to Roger M. Herman,rmh@phys.psu.edu
}

Received 27 April 2009; Accepted 19 July 2009

Academic Editor: Roland Stamm

Copyright () 2010 Roger M. Herman. This is an open access article distributed under the Creative Commons Attribution License, which permits unrestricted use, distribution, and reproduction in any medium, provided the original work is properly cited.

A dipole correlation function which incorporates velocity-changing (motional narrowing) effects and the effects of speeddependent Lorentz relaxation rates into otherwise Voigt profile correlation functions is developed, based partly upon previous work by the author. For the first time simple closed expressions, which lend themselves to elementary calculation beginning only with the relevant parts of intermolecular interaction energies, are developed for the cubic time-dependent term within the exponent describing the decay of the correlation function. This term is of first order in perturber number density, as are the Lorentz parameters, and is complex, thereby allowing for narrowing, changing in shape and asymmetry in the line profile. "Soft" and "hard" collisions play no explicit role, though both are variously present for each line. Quartic time dependencies are also discussed, though they are thought to be negligible in nonhydrogen molecular spectroscopy. Finally, some comments are added about a relevant technique for hydrogen spectra.

\section{Introduction}

For several decades, now, neutral species coherence transport and relaxation has followed a number major thrusts, above and beyond the simplest convolution of the optical coherence interruption (Lorentz) and Gaussian (Doppler) contours, as embodied in the Voigt profile for isolated spectral lines. To mention a few, five such developments come immediately to mind: (1) the incorporation of the speed dependence of coherence destruction (with speed dependent Lorentz lineshape parameters) within the Voigt convolution (SDVP effect) [1-3]; (2) the incorporation of collisional reduction of free-streaming molecular motions, which had previously been assumed in the initial inclusion of Doppler broadening, known as Dicke (or motional) narrowing [4]; (3) finite duration of collision effects [5] as they influence narrow lines that otherwise are regarded as having been generated by effectively instantaneous collisions; (4) line-mixing effects, in which coherent collisional mixing of the transition of immediate interest with other nearby (frequency-wise) transitions leads to an effective blending of contours in which each becomes distorted into combination Lorentziananomalous dispersion (Fano) contour [5-7]; (5) nonlinear spectroscopies [8], where, in two-photon absorption, the Doppler broadening may be cancelled out, due to the opposite propagation direction (and therefore compensatory momentum uptake by the excited molecule) of the two photons involved in producing the absorption signals. Also, in saturation spectroscopies, a primary interaction causes a population "hole" to be produced at a selected frequency corresponding to a specific Doppler velocity group, which can then be broadened directly by velocity-changing collisions for detection by a secondary beam. These are what might be called "special effects" which lie outside of the scope of this article, although the methods described herein are sometimes useful in dealing with these phenomena [9]. Line mixing effects can be readily introduced into the formalism that will be described, though they will again be omitted from the present discussion, for simplicity. Finite duration effects fall into two categories, the first being that for a single spectroscopic line the correlation function for nearly zero time (equal to or less than the duration of typical collisions) will differ from the more typical decaying exponential time dependence seen in the longer time behavior. This gives rise to a short time correction to the correlation function which leads to the appearance of an additive (as opposed 
to multiplicative as in some literature; see [10], e.g.) low intensity, broad frequency contribution that will ensure finite $n$th moments of the intensity distributions for all $n$, as must result for analytic forms of the correlation functions. [1114] The second finite-duration effect would be described as true binary collisions throughout the collision sequences which give rise to Lorentzian shapes. These would primarily lead to slight quadratic (in density) Lorentzian width and shift dependences. The finite duration-of-collision effects are indeed important for the accurate description of narrow isolated spectral features. Nonetheless, for purposes of simplicity, they again will be omitted from further discussion in the present theoretical treatment.

Of special interest in the present paper are the highly detailed experimental studies of individual spectral lines, such as those carried out by Pine et al. [15-17], and Wehr $[18,19]$ for hydrogen halide spectra, by Rohart et al. [20, 21] for atmospheric ozone, by Lisak et al. [22] for water vapor and by Priem et al. [23] and Wehr et al. [24, 25] for CO. Self- and foreign gas-perturbed hydrogen Raman spectra $[26,27]$ show effects related to those cases mentioned above, though the analysis of the latter lies a bit outside of the present development. Most of the articles cited contain theoretical analyses and there exist many independent theoretical analyses, too numerous to cite here. In the related analysis, there have emerged two models that seem, in varying situations, to be applicable to an understanding of the experimental results: the "soft" collision model first introduced by Galatry [10] in which, in collisions leading to internal state coherence modification, collisions gently act to reduce the free-streaming motions of molecules as assumed in Doppler broadening, together with the introduction of diffusional translational motions, and the "hard" collision model first introduced by Rautian and Sobel'man [28], in which, in addition to internal state coherence modification, molecular motion is totally randomized to the extent allowed by dynamical constraints. To a large extent these models are still in use, though some efforts have been made to bridge the gaps between the two pictures through the introduction of an additional hardness parameter. In actuality, both pictures operate separately but simultaneously, to a greater or lesser extent in producing any given spectral line, in that the distant collisions, which traditionally do not substantially limit internal state coherence but are often the primary cause of line shifts would typically be characterized as being soft, only slightly altering translational motions in any of the numerous such encounters, while the hard collisions would accompany events in which the coherence is often nearly terminated. In the present paper, we will introduce a treatment which formally makes no delineation between the soft and hard collision cases, including both types in a "seamless" unified description of all of the abovementioned processes. Also included in a natural way are all aspects of interference between internal state coherence limitation and velocity changes, which can give rise to slight asymmetries of the lines which have, on occasion, been observed.

One of the difficulties of the descriptions of Dicke narrowing is that there seem not to be any reasonably transparent first principles expressions, or series of expressions giving the relevant parameters associated with these phenomena. In the present paper we shall, for the first time, derive intuitively appealing closed expressions for the relevant quantities, in terms of relevant parts of intermolecular potential functions and intrinsic parameters such as number density and temperature. The present work follows a partial description which was first published in 2000 [29], which carried the capricious title utilizing the phrase "Theory for Everything..." henceforth referred to as paper I. However, the detailed expressions for relevant parameters occurring in the correlation functions are newly derived in the present paper. While the closely allied SDVP effects have been variously discussed, the first description in which it was systematically related to the time dependence of the correlation function was provided by Looney [30], and that treatment will be followed here, with modifications.

The dipole-dipole (or other optically active operators, depending upon the spectroscopy being described) correlation function for an isolated transition can be described as having an exponential behavior of type [30, 31]:

$$
\exp \left[-\gamma t-\frac{k_{B} T}{2 m} k^{2} t^{2}+\beta t^{3}-\delta t^{4}+\cdots\right], \quad t>0 .
$$

In the first term $\gamma=\gamma_{r}+i \gamma_{i}$ represents the standard internal state coherence interruption effect, providing a Lorentz shape half-width at half maximum, $\gamma_{r}$, and line shift, $-\gamma_{i}$. The second term expresses the Doppler effect which, by itself, leads to the standard Gaussian lineshape with $k$ being the optical wavevector magnitude. The cubic term depends upon the coefficient $\beta$, which we might call the first motional narrowing coefficient. It is intrinsically complex, due to correlations of internal phase perturbation and translational velocity changes. Then $\delta$ is the second motional narrowing coefficient, and so on. We will supply closed expressions for ready calculation of $\beta$, and indicate how one may approach the calculation of $\delta$, in the unlikely event that it would be needed. Higher order terms are required to give a convergent expression for $C(t)$ at very large times, opposing the indefinite increase in the cubic term. But explicit calculation would only proceed to the point that $C(t)$ becomes essentially zero, even with only the cubic term, and the higher terms would remain as being negligible, at which point the Fourier transform integration would be truncated. Higher parameters are of unlikely practical significance in virtually all nonhydrogen spectroscopy. In hydrogen spectroscopy (through Raman, quadrupole or permanent and collisionally induced dipole sharp line spectra in HD) motional narrowing is extremely important $[26,27]$ at moderately low densities (corresponding to around one to ten atmospheres at room temperature). For these cases the above-mentioned higher terms become important. While the present theory surely applies (in a different limit) to these cases, detailed studies will not be carried out at this time. Nonetheless, an indication of how one might approach the molecular hydrogen problem is suggested.

We will assume that classical paths are sufficient for the description of collisional processes. As indicated, one can 
presumably make the entire calculation quantum mechanically as has been done in the past for the calculation of Lorentz lineshape parameters [32]. But there are other possibilities: One might accept fully quantum computation for the Lorentz parameters, accept as true in quantum mechanics as well as classical mechanics the simple Doppler terms, and use classical methods for the description of motional narrowing. If ones interest is solely to understand motional narrowing, measured values of the Lorentz parameters can be accepted, while ensuring that the correct density dependences are strictly adhered to throughout.

A note of caution should be expressed at the present time. This is, that obtaining agreement between theory and experiment on a single molecular line at a single density can lead to deceptive satisfaction. One must strive to analyze all lines that have been observed at all possible pressures, while rigorously adhering to the correct pressure dependencies of the real and imaginary parts of $\gamma$ and $\beta$ throughout.

\section{Theoretical Considerations}

The development of a correlation function describing isolated spectral lines, omitting duration-of-collision effects, essentially follows that of paper I. Minor changes will be made, to more accurately reflect current notation. In particular, the subscripts " $f$ " will be dropped from expressions involving the correlation function, the choice of final and initial states in the transition being obvious. Moreover, $\tau$ and $t$ will be replaced by $t, t^{\prime}$, and $\mathbf{v}_{\mathbf{o}}, \mathbf{v}$ will be replaced by $\mathbf{v}, \mathbf{v}^{\prime}$, respectively. Vectors will be denoted by boldface type.

The spectral line profile for absorption from a lower state $i$ to upper state $f$ (normalized to the relevant dipole moment matrix element squared) can be represented by the form

$$
\mathcal{L}_{f i}(w)=\frac{\left|\mu_{f i}\right|^{2}}{\pi} \operatorname{Re} \int_{0}^{\infty} C_{f i}(t) \exp \left[\left(w_{f i}-w\right) t\right] d t
$$

where $C_{f i}(t)$, the correlation function, satisfies the ensemble average:

$$
C_{f i}(t) \equiv C(t)=\left\{U_{f f}^{*}(t) U_{i i}(t) \exp [i \mathbf{k} \cdot \mathbf{R}(t)]\right\}_{\text {ens.avg.' }}
$$

where $\mathbf{k}$ is the optical propagation vector, $\mathbf{R}$ is the molecular displacement following time $t$ relative to the position at time $t=0$, and $U_{f f}$ and $U_{i i}$ are diagonal time-development matrix elements for the respective internal molecular states resulting from whatever collisional events may occur in the time interval $0 \rightarrow t$. As indicated above, classical paths are envisioned in the present description, though quantum mechanical descriptions of the translational motion problem can be readily developed. For the internal states, $U_{f f}^{*}$ and $U_{i i}$ can be written as product matrix elements for noncorrelated random collisions. However, the same factorization cannot be made for the translational factor. And it is this nonMarkovian property that has possibly inhibited the development which now is presented here. Concentrating on the internal states, for a single collision it is well known $[33,34]$ that the product of $U$ matrix elements can be put into the form

$$
\begin{aligned}
& S(\mathbf{v} ; \mathbf{b}, \mathbf{u})= \exp \left[-i \eta_{f i}(\mathbf{v} ; \mathbf{b}, \mathbf{u})\right] \\
& \times\left(1-S_{f}^{*^{(2)}}(\mathbf{v} ; \mathbf{b}, \mathbf{u})-S_{i}^{(2)}(\mathbf{v} ; \mathbf{b}, \mathbf{u})\right. \\
&\left.\quad+S_{f}^{*^{(4)}}+S_{i}^{(4)}+\cdots\right),
\end{aligned}
$$

where the superscripts (2), (4) indicate the order of collisional inelastic perturbation. The phase shift term can be represented quite exactly to all orders of perturbation [35] while the inelastic terms, $S_{f, i}^{(n)}$, often are subject to various mathematical approximations as the strength of the collisional interruption increases [36]. While the ATC treatment $[33,34]$ had envisioned the $S_{f, i}^{(n)}$ terms as being real, they are in general complex, at times contributing significantly to line shifts, otherwise given solely by the phase shifts [37-39].

In the present work, we will regard the calculation of the collisional interruption quantities $S(\mathbf{v} ; \mathbf{b}, \mathbf{u})$ as being completely settled, along with the calculation of outgoing velocities following collisions. We will indicate such dynamical processes as leading to post-collisional velocities $\mathbf{v}^{\prime}(\mathbf{v} ; \mathbf{b}, \mathbf{u})$. Here $\mathbf{v}$ is the input velocity of the optically active molecule, $\mathbf{b}$ being the vector impact parameter lying in the plane perpendicular to $(\mathbf{v}-\mathbf{u})$, with $\mathbf{u}$ being the perturber initial velocity in any particular encounter.

Following the development of paper $I$, we now think in terms of a collision as being one in which $\mathbf{b}$ lies within $\mathbf{a}$ somewhat large radius of collision $B$, in an attempt to think about probabilities for zero collisions and a time distribution for a first collision. The actual value of $B$ is unimportant, and it will be seen to fall away from the description as the theory is developed. For now, in the interest of simplicity, let us envision the perturbers as being motionless. According to Poisson statistics, for collisions within radius $B$ the probability for there being no collisions in the interval $0 \rightarrow t$ is simply

$$
\exp \left[-N \bar{v} \pi B^{2} t\right]
$$

while the probability per unit time of a first collision is

$$
\frac{d P_{1}^{(t)}}{d t}=-\frac{d P_{0}^{(t)}}{d t}=N \bar{v} \pi B^{2} \exp \left[-N \bar{v} \pi B^{2} t\right],
$$

with $N$ being the perturber number density. Of course, following the first collision there can be a multitude of collisional events, though they will be included implicitly in the appearance of correlation functions for times following the first collision. For a specific initial velocity $v$, in the absence of any collision, the correlation function will simply be a translational (free-streaming) exponential $\exp [i \mathbf{k} \cdot \mathbf{v} t]$, so that $C(\mathbf{v} ; t)$ will have a no-collision contribution:

$$
\exp \left[-N \bar{v} \pi B^{2} t+i \mathbf{k} \cdot \mathbf{v} t\right] .
$$

But now, in addition, we must include the first collision contribution, distributed throughout the interval $0 \rightarrow t$ :

$$
\int_{0}^{t} N \bar{v} \pi B^{2} d t^{\prime} \exp \left[-N \bar{v} \pi B^{2} t^{\prime}\right] S(\mathbf{v}) C\left(\mathbf{v}^{\prime} ;\left(t-t^{\prime}\right)\right),
$$


where $S$ contains the collisional interruption effects, and $\mathbf{v}^{\prime}$ represents the outgoing velocity in this first single collision. Of course one has to specify the actual impact parameter integration within $B$, and the integration on $\mathbf{u}$, utilizing the Maxwell distribution of perturber velocities $f(\mathbf{u})$, with the result

$$
\begin{aligned}
C(\mathbf{v} ; t)= & \exp \left[-N \bar{v} \pi B^{2} t+i \mathbf{k} \cdot \mathbf{v} t\right] \\
& +N \int_{0}^{t} d t^{\prime} \exp \left[-N \bar{v} \pi B^{2} t^{\prime}+i \mathbf{k} \cdot \mathbf{v} t^{\prime}\right] \\
& \times \int_{0}^{B} d \mathbf{b} \int d \mathbf{u} f(\mathbf{u})|\mathbf{v}-\mathbf{u}| S(\mathbf{v} ; \mathbf{b}, \mathbf{u}) C\left(\mathbf{v}^{\prime} ;\left(t-t^{\prime}\right)\right)
\end{aligned}
$$

The integrations over $\mathbf{b}$ and $\mathbf{u}$ are two- and threedimensional, respectively. This equation contains the fundamental idea of this paper. As yet, however, this equation is somewhat difficult to utilize in a practical way, and the problem of how to specify $B$ remains unsolved. These difficulties can be remedied through the transformations indicated in $I$ and included in this paper, in the appendix. The final equation from the appendix, which has eliminated the troublesome feature of specifying $B$ and which is capable of yielding fundamental expressions for the line shape parameters of current interest is repeated here:

$$
\begin{aligned}
C(\mathbf{v} ; t)=\exp [ & i \mathbf{k} \cdot \mathbf{v} t-N \int_{0}^{t} d t^{\prime} \int_{0}^{\infty} d \mathbf{b} \int d \mathbf{u} \\
& \left.\times f(\mathbf{u})|\mathbf{v}-\mathbf{u}|\left(1-S(\mathbf{v} ; \mathbf{b}, \mathbf{u}) \frac{C\left(\mathbf{v}^{\prime} ; t^{\prime}\right)}{C\left(\mathbf{v} ; t^{\prime}\right)}\right)\right]
\end{aligned}
$$

keeping in mind that $\mathbf{v}^{\prime}=\mathbf{v}^{\prime}(\mathbf{v} ; \mathbf{b}, \mathbf{u})$ in accordance with classical dynamics throughout. Note that this equation is equivalent to (10) of paper $I$.

Over the years, as mentioned above, the collision efficiency function $S(\mathbf{v} ; \mathbf{b u})$ has been the subject of traditional line shape studies within the classical path formalism. More recently quantum mechanical treatments of the translational motions have been included [32], with considerable success. Within the present theoretical development it is difficult to see how to include quantum translational motions because of the difficulty of computing differential cross-sections for internal state disruption.

\section{Applications}

The beauties of (10) are twofold. The first is purely conceptual, in that $C(\mathbf{v} ; t)$ follows all possible collisional histories, with many possible original and intermediate velocities, for ending at time $t$ having velocity $\mathbf{v}$. But also, by redefining the various time intervals and utilizing the principle of detailed balance, by which the $(\mathbf{v}, \mathbf{u}) \rightarrow\left(\mathbf{v}^{\prime}, \mathbf{u}^{\prime}\right)$ process is equally likely as its reverse process, one can see that $C(\mathbf{v} ; t)$ also can be viewed as applying to all histories such that one begins at $t=0$ with velocity $\mathbf{v}$ and ends up with the reverse array of intermediate and final velocity $\mathbf{v}^{\prime}$. The second is the more practical aspect of how to apply this equation to finding correlation functions that are useful in describing the wealth of spectra that can be illustrative of the effects of collisional narrowing. To accomplish this, we will explicitly find the complex $t^{3}$-dependent term in the correlation function in a closed form that represents such an expression for the first time. In addition, we will point the reader in a direction such as to be able to recover the $t^{4}$ terms, if the need should arise. We propose using an iterative technique, in which (10) will be solved in successive orders of refinement, such that the first nonVoigt term will be linear in number density of perturbers, the second will be quadratic, and so on. To begin with, let us rewrite (10) in the alternate form

$$
\begin{aligned}
& C(\mathbf{v} ; t) \\
& \begin{aligned}
= & \exp [i \mathbf{k} \cdot \mathbf{v} t] \\
\times \exp & {\left[-N \int_{0}^{t} d t^{\prime} \iint d \mathbf{b} d \mathbf{u}|\mathbf{v}-\mathbf{u}|\right.} \\
& \left.\times\left((1-S(\mathbf{v} ; \mathbf{b}, \mathbf{u}))+S(\mathbf{v} ; \mathbf{b}, \mathbf{u})\left(1-\frac{C\left(\mathbf{v}^{\prime}, t^{\prime}\right)}{C\left(\mathbf{v}, t^{\prime}\right)}\right)\right)\right] .
\end{aligned}
\end{aligned}
$$

But the $(1-S(\mathbf{v} ; \mathbf{b}, \mathbf{u}))$ term in this equation, by itself, is simply the ordinary pressure broadening, thus

$$
\begin{aligned}
C(\mathbf{v} ; t)=\exp [ & -\gamma t+i \mathbf{k} \cdot \mathbf{v} t-N \int_{0}^{t} d t^{\prime} \iint d \mathbf{b} d \mathbf{u} \\
& \left.\times|\mathbf{v}-\mathbf{u}| S(\mathbf{v} ; \mathbf{b}, \mathbf{u})\left(1-\frac{C\left(\mathbf{v}^{\prime} ; t^{\prime}\right)}{C\left(\mathbf{v} ; t^{\prime}\right)}\right)\right] .
\end{aligned}
$$

The third term in the exponent represents the complete effect of motional narrowing. Denoting $C^{(1)}(\mathbf{v} ; t)$ as a first iteration correlation function, we now use the collisionless form of the ratio of $C$ 's shown in (12) to be $\exp \left[-i k \cdot\left(\mathbf{v}-\mathbf{v}^{\prime}\right) \mathbf{t}^{\prime}\right]$ to obtain

$$
-N \iint d \mathbf{b} d \mathbf{u}|\mathbf{v}-\mathbf{u}| S(\mathbf{v} ; \mathbf{b}, \mathbf{u}) t\left(1+\frac{\exp \left[-i \mathbf{k} \cdot\left(\mathbf{v}-\mathbf{v}^{\prime}\right) t\right]-1}{i \mathbf{k} \cdot\left(\mathbf{v}-\mathbf{v}^{\prime}\right) t}\right)
$$

following integration on $t^{\prime}$, as the motional narrowing exponent. In Taylor expansion form, this is

$$
\begin{aligned}
- & N \iint d \mathbf{b} d \mathbf{u}|\mathbf{v}-\mathbf{u}| S(\mathbf{v} ; \mathbf{b}, \mathbf{u}) \\
& \times\left(\frac{i \mathbf{k} \cdot\left(\mathbf{v}-\mathbf{v}^{\prime}\right) t^{2}}{2}+\frac{k^{2}\left(v_{z}-v_{z}^{\prime}\right)^{2} t^{3}}{6}+\cdots\right)
\end{aligned}
$$

representing the lowest order motional narrowing terms which should, in all cases, be computer friendly and which, once computed, should allow one to find $C(t)$ through the $t^{3}$ motional narrowing. There are $t^{3}$ terms in $C(t)$ arising from speed dependent Voigt profile effects, however, as will be seen in the next section.

It is not so easy to proceed further from this point without (elementary) computer analysis. Nonetheless, we can make arguments which may indicate some of the basic properties. Beginning with the first Taylor term we note that integrating only over the angles for $\mathbf{b}$ and $\mathbf{u}$, the average velocity difference $\left(\mathbf{v}-\mathbf{v}^{\prime}\right)$ must take the direction of $\mathbf{v}$ itself. 
Therefore, this part of the exponent can be written in the form $\exp \left[-i \mathbf{k} \cdot \mathbf{v} X t^{2}\right]$, with

$$
X=\left(\frac{N}{2}\right) \iint d \mathbf{b} d \mathbf{u}|\mathbf{v}-\mathbf{u}| S(\mathbf{v} ; \mathbf{b}, \mathbf{u})\left(1-\frac{\mathbf{v} \cdot \mathbf{v}^{\prime}}{v^{2}}\right)
$$

which is first order in perturber number density $N$. Although $X$ in the above expression probably varies with speed, in general this will be a small effect compared r.m.s. value of speed effect (and, indeed, with the speed dependence of $\gamma$ itself, which will be addressed below).

At this stage, therefore,

$$
C(\mathbf{v} ; t) \cong \exp \left[-\gamma t+i \mathbf{k} \cdot \mathbf{v}\left(t-X t^{2}\right)\right] .
$$

From (15) it is seen that $X$ is complex, with the imaginary part of $\beta$ in $C(t)$ ultimately arising from an interference between internal collisional phase shifts and velocity $(z$ component) changes.

The second term in (14), is less obvious in its effects, and the averaging over $\mathbf{b}$ and $\mathbf{v}$ angles is more subtle. The average will depend upon $v_{x}$ and $v_{y}$, as well as $v_{z}$. As such, this term explicitly handles Doppler effects following collision arising from the production of $v_{z}$ components generated from all velocity components present at $t=0$. The overall effect of such terms might be quite small, however, as can be seen by reference to typical situations. For collisions between hydrogen halide and other molecules of somewhat similar mass, for the longer range encounters, $\mathbf{v}$ will hardly change, leaving $\left(\mathbf{v}-\mathbf{v}^{\prime}\right)$ as a very small quantity; for the closer collisions that can indeed change $v$ through scattering more toward right angles, for which $\left(\mathbf{v}-\mathbf{v}^{\prime}\right)$ may be quite large, the collisional interruption given by $S(\mathbf{v} ; \mathbf{b}, \mathbf{u})$ may be so large as to effectively screen the dipole moments from being sensitive to these velocity changes. Of course, for He being the foreign gas, large angle scattering of the optically active molecule will not be much of a factor, leaving $\left(\mathbf{v}-\mathbf{v}^{\prime}\right)$ as a very small term. Similar arguments might hold for the term linear in $\left(\mathbf{v}-\mathbf{v}^{\prime}\right)$ analyzed above, but in view of its first order appearance this term might remain the principal source of the $t^{3}$ term. To address this question for either case, let us imagine that the smaller angle scattering is most likely to survive the coherence loss dictated by $S(\mathbf{v} ; \mathbf{b}, \mathbf{u})$. Thus, for polar and azimuthal scattering angles $\psi, \chi$, measured form the incident velocity $\mathbf{v}$ direction, the term $(1 / 2) \mathbf{k} \cdot\left(\mathbf{v}-\mathbf{v}^{\prime}\right) t^{2}$ would be proportional to $(1 / 2) k v_{z}(1-\cos \psi) t^{2}$, and for small angles, $(1 / 4) k v_{z} \psi^{2} t^{2}$ prior to averaging over $\psi$. Relative to this, the term $(1 / 6) k^{2}\left(v_{z}-v_{z^{\prime}}\right)^{2} t^{3}$ is proportional to $\left(k^{2} / 6\right)\left(v_{z}^{2}(1-\right.$ $\left.\cos \psi)^{2}+\left(v_{x}^{2}+v_{y}^{2}\right) \sin ^{2} \psi \cos ^{2} \chi\right)$. One immediately averages over $\chi$; the first term is quartic in the small scattering angle $\psi$, and can therefore be ignored. (This shows that the major effect in these terms comes from the $v_{x}, v_{y}$ components of $\mathbf{v}$ being collisionally diverted toward the $\pm z$-directions.) Therefore this term is approximately equal to $\left(-k^{2} / 6\right)\left(\left(v_{x}^{2}+v_{y}^{2}\right) / 2\right) \psi^{2} t^{3}$. This suggests that the entire exponent for this term can be written as

$$
\begin{gathered}
\frac{-N}{6} k^{2} t^{3} \iint d \mathbf{b} d \mathbf{u} f(\mathbf{u})|\mathbf{v}-\mathbf{u}| S(\mathbf{v} ; \mathbf{b u})\left(v_{z}-v_{z}^{\prime}\right)^{2} \\
=-k^{2}\left(\frac{\left\langle v_{x}^{2}+v_{y}^{2}\right\rangle}{2}\right) Y t^{3}
\end{gathered}
$$

with

$$
Y=\frac{N}{6} \iint d \mathbf{b} d \mathbf{u} f(\mathbf{u})|\mathbf{v}-\mathbf{u}| S(\mathbf{v} ; \mathbf{b u}) \frac{2\left(v_{z}-v_{z}^{\prime}\right)^{2}}{\left\langle v_{x}^{2}+v_{y}^{2}\right\rangle}
$$

the factor 2 somewhat compensates the $\cos ^{2} \chi$ averaging. In this form, $Y$ will tend to be insensitive to temperature, and is proportional to $N$.

The overall correlation function $C(t)$ is now found upon averaging over $v_{z}$, in the form

$$
\begin{aligned}
C(t)= & \sqrt{\frac{m}{2 \pi k_{B} T}} \int d v_{z} \\
& \times \exp \left[-\gamma t-\frac{m v_{z}^{2}}{2 k_{B} T}+i k v_{z} t(1-X t)-\frac{k^{2}\left\langle v_{x}^{2}+v_{y}^{2}\right\rangle}{2} Y t^{3}\right] \\
= & \exp \left[-\gamma t-\left(\frac{k_{B} T}{2 m}\right) k^{2} t^{2}(1-X t)^{2}-\left(\frac{k_{B} T}{m}\right) k^{2} Y t^{3}\right]
\end{aligned}
$$

noting that $\left\langle v_{x}^{2}+v_{y}^{2}\right\rangle=2 k_{B} T / m$. The $t^{4}$-term is quadratic in $N$. By itself it gives an incomplete accounting of the entire $t^{4}$ dependence so we will, for present purposes, work only to order $t^{3}$, for which

$$
C(t)=\exp \left[-\gamma t-\left(\frac{k_{B} T}{2 m}\right) k^{2} t^{2}+\frac{k_{B} T}{m} k^{2}(X-Y) t^{3}\right] .
$$

Comparison of small angle approximations reveals that $Y$ is about equal to $2 X / 3$, tending to diminish the motional narrowing. It actually represents a motional-broadening effect associated with collisional production of $v_{z}$ velocity components (from previously $v_{y}$ and $v_{y}$ components), as noted. It is seen that both $X$ and $Y$ are complex quantities, the imaginary parts leading to asymmetries in line shapes; some of which have been observed.

For many spectra the fourth order, $t^{4}$ terms in the exponent of $C(t)$ are entirely negligible. If they are needed they will arise from the neglected $t^{4}$ terms as noted above, together with the second iteration $C^{(2)}$ in which the results of the first iteration for $C^{(1)}(\mathbf{v} ; t)$ are included. The calculation will follow the lines already set out, here, for $C^{(1)}(\mathbf{v} ; t)$, leading to further integrations, for pairs of collisions, in which not only $(\mathbf{v}, \mathbf{u}) \rightarrow \mathbf{v}^{\prime}$, but also $\left(\mathbf{v}^{\prime}, \mathbf{u}^{\prime}\right) \rightarrow \mathbf{v}^{\prime \prime}$. To work this out in detail is straightforward, though this lies beyond the scope of the present paper. In addition, there is a (neglected) $t^{4}$ term found in the next section.

Finally, as previously noted, hydrogen spectra are extremely collisionally narrowed, such that an expansion in terms of individually computed powers of $t$ would seem pointless. In this case, it might be possible to use a model for the collisionally narrowed $C(\mathbf{v} ; t)$ for all $\mathbf{v}$, then for purposes of calculating a next iteration result, insert the model forms into (10) for use in the ratio $C\left(\mathbf{v}^{\prime} ; t\right) / C(\mathbf{v} ; t)$. In this manner one can recover a once-refined result for $C(\mathbf{v} ; t)$ for all $\mathbf{v}$ and $t$. To the extent that the initial guess was reliable, the corrections within the first refinement result will indeed be small. This would then lead to correlation functions which can then be averaged over $\mathbf{v}$ leading to a refined expression for $C(t)$ which in turn can be used directly to obtain the line profile. 


\section{Speed Dependent Voigt Profile Effects}

For some time now it has been known that the speeddependence of internal coherence relaxation has had importance in determining the shape of spectral lines, shifting them away from the simple Voigt profile $[1-3,23]$. Because this lies outside of the major thrust of the present paper we will only sketch the manner in which these effects can provide time-dependent terms in the correlation function $C(t)$. This, again, follows the development of Looney [30]. Beginning with the form of $C(\mathbf{v} ; t)$ leading to the voigt profile, one can make simple changes that will lead to the appropriate time dependences in the exponential decay rate for $C(\mathbf{v} ; t)$. Let us begin with the simple form of $C(\mathbf{v} ; t)$, modified so as to include velocity dependences of $\gamma$. Let $\gamma(v)$ depend upon the speed of the optically active molecule in the following manner:

$$
\gamma(v)=\gamma_{o}+\left(\frac{d \gamma}{d\left(v^{2}\right)}\right)_{N}\left(v^{2}-\left\langle v^{2}\right\rangle\right)+\cdots
$$

as a general expression, $\left\langle v^{2}\right\rangle$ being the value for the mean squared speed, about which we will expand. Subscript $N$ is used to remind us that this derivative is to be considered for constant number density. For present purposes we work within the quadratic approximation, for which we can write

$$
\gamma(v)=\gamma_{o}+\gamma^{\prime}\left(v_{x}^{2}-\left\langle v_{x}^{2}\right\rangle+\cdots y, z\right)
$$

with $\gamma^{\prime}=\left(d \gamma / d v^{2}\right)_{N}$. The averages over velocity components are easily accomplished, ignoring, for the moment, the $\exp \left[-\gamma_{0} t\right]$ term. We begin with the average over $v_{x}$, using the Maxwell distribution, such that

$$
C_{x}(t)=\sqrt{\frac{m}{2 \pi k_{B} T}} \exp \left[-\frac{m v_{x}^{2}}{2 k_{B} T}-\gamma^{\prime}\left(v_{x}^{2}-\left\langle v_{x}^{2}\right\rangle\right) t\right]
$$

$k_{B}$ being the Boltzmann constant and $m$ the mass of the optically active molecule. We define the constant

$$
Z=\frac{2 \gamma^{\prime} k_{B} T}{m}
$$

for notational simplicity, so that $C_{x}(t)$ can be readily obtained, in the form

$$
C_{x}(t)=(1+Z t)^{-1 / 2} \exp \left[\gamma^{\prime}\left\langle v_{x}^{2}\right\rangle t\right] .
$$

By noting that $\left\langle v_{x}^{2}\right\rangle=k_{B} T / m$, one can find through expansion and subsequent identification of terms

$$
\begin{aligned}
C_{x}(t)= & \left(1-\frac{1}{2} Z t+\frac{3}{8} Z^{2} t^{2}-\frac{5}{16} Z^{3} t^{3}+\cdots\right) \\
& \times\left(1+\frac{1}{2} Z t+\frac{1}{8} Z^{2} t^{2}+\frac{1}{48} Z^{3} t^{3}+\cdots\right) \\
\cong & \left(1+\frac{1}{4} Z^{2} t^{2}-\frac{1}{6} Z^{3} t^{3}\right)
\end{aligned}
$$

which is exact to terms in $t^{3}$, provided that $\bmod |Z t|<1$. This will always be true for short enough times, and our assumption is that it will be possible to do this for any physically realistic times.
The same steps can be followed for the $y$-integration, as well. With the $z$ integration, we begin with the same integral, but now with the Doppler (translational) phase factor, $\exp \left[i k v_{z}\right]$, leading directly to the result

$$
\begin{aligned}
C_{z}(t)= & \sqrt{\frac{m}{2 \pi k_{B} T}} \exp \left[\frac{Z t}{2}\right] \\
& \times \int d v_{z} \exp \left[-\frac{m v_{z}^{2}}{2 k_{B} T}(1+Z t)+i k v_{z} t\right] \\
= & (1+Z t)^{-1 / 2} \exp \left[\frac{Z t}{2}-\frac{k_{B} T k^{2} t^{2}}{2 m(1+Z t)}\right] .
\end{aligned}
$$

Within terms to order $t^{3}$, this is equivalent to the form

$$
C_{z}(t)=\exp \left[-\left(\frac{k_{B} T k^{2}}{2 m}-\frac{1}{4} Z^{2}\right) t^{2}+\left(\frac{k_{B} T k^{2} Z}{2 m}-\frac{1}{2} Z^{3}\right) t^{3}\right] .
$$

Note that $Z$ is proportional to number density, so that the simple Doppler distribution will be altered (in width, e.g.) by terms quadratic in density, while the motional narrowing term will contain parts linear, and varying as the third power in density. While the change in the simple Doppler correlation function will probably be beyond detection, we will retain that term for reference, while dropping the $Z^{3}$ term. By now multiplying the $x$-, $y$ - and $z$-results, and recovering the $\exp \left[-y_{0} t\right]$ factor, we find

$$
C_{\mathrm{SDVP}}(t)=\exp \left[-\gamma_{o} t-\left(\frac{k_{B} T k^{2}}{2 m}-\frac{3 Z^{2}}{4}\right) t^{2}+\frac{k_{B} T k^{2} Z}{2 m} t^{3}\right] .
$$

This represents the Voigt correlation function, whose width is supplemented the small (usually negligible) $Z^{2} t^{2}$ term and by a further exponential $t^{3}$ term. While $\gamma^{\prime}$, and therefore $Z$ are presumably complex, the speed dependence of line shifts is thought to be minimal, because the increase in collision rate will be roughly matched by corresponding decreases in phase shifts, tending toward cancelation of the speed dependence. Therefore, we will assume $Z$ to be real.

As described by Looney [30], the value of $\gamma^{\prime}$ can be inferred from the temperature dependence of $\gamma$, provided that it is known. To see this, note that

$$
\gamma^{\prime}=\left(\frac{d \gamma}{d\left(v^{2}\right)}\right)_{N}=\frac{d T}{d\left(v^{2}\right)}\left(\frac{d \gamma}{d T}\right)_{N}=\frac{m}{3 k_{B}}\left(\frac{d \gamma}{d T}\right)_{N} .
$$

This holds only if the perturbers are motionless (i.e., have infinite mass). For finite mass perturbers, however, the relative collision speeds will increase more rapidly with temperature, and $m$ must be replaced by the reduced mass of the collision pair, with the result

$$
\gamma^{\prime}=\frac{m m_{x}}{3\left(m+m_{x}\right) k_{B}}\left(\frac{d \gamma}{d T}\right)_{N}
$$

with $m_{x}$ being the mass of the perturbing molecule. This will obviously be most valuable if the temperature dependence of $\gamma$ is known from separate measurements. 


\section{Conclusions}

As a result of the above analysis the $t^{3}$ terms in the correlation function are given by the contributions coming from velocity-changing collisions (cf. (20)) together with the above SDVP effect, equation (29), giving as a final result

$$
\beta=\frac{k_{B} T}{m} k^{2}\left(X-Y+\frac{Z}{2}\right)
$$

with $X, Y$, and $Z$ being given by (15), (18), and (24). Each term is linear in perturber number density $N$.

To summarize, a formalism has been presented whereby one can find the relevant terms for the dipole-dipole correlation function needed to characterize the line shapes in the impact approximation for isolated (atomic or) molecular lines perturbed by foreign gases. Through standard techniques this formalism could be extended to a fully quantum description of the entirety of the collision problem treated here. In addition, one could treat the problem of line-mixing with neighboring transitions within the present formalism. For purposes of clarity, neither of these steps is presented here. The cubic time-dependent terms of the exponent describing the exponential decay of the correlation function have been explicitly found in terms of averages utilizing only equilibrium velocity distributions and the variously important terms of the perturber interaction with the optically active molecule. Although both "soft" and "hard" types of collisions occur for various impact parameters for almost every line, the present theory makes no fundamental distinction between these limits, and makes a seamless transition from one to the other type of behavior.

For the velocity changing $t^{3}$ effects there exist two competing influences: A line narrowing due to the fact that higher speed components tend to be reduced in collisions and a line broadening associated with collisional production of velocity components parallel to the optical propagation vector, derived from pre-collision components perpendicular to this direction. The two effects oppose one another, with the broadening being about two thirds of the narrowing. This means that the velocity-change broadening will not dominate the narrowing, but at the same time it is substantial. In addition, speed-dependent Voigt profile effects are active in giving rise to a further $t^{3}$ narrowing exponential term (presuming that the speed dependence of the ordinary Lorentz lineshape parameters shows an increase with increasing velocity, which is the standard case).

In hydrogen molecular spectra the coherence limiting effects are very small. Accordingly one can, at some pressures, see a pronounced line narrowing. One might approach the theoretical problem by using the best model form of $C(\mathbf{v} ; t)$ (based upon a Dicke type approach), then compute $C(\mathbf{v} ; t)$ using (10), with the ratio $C\left(\mathbf{v} ; t^{\prime}\right) / C\left(\mathbf{v}^{\prime} ; t^{\prime}\right)$ replaced by the model result. $C(\mathbf{v} ; t)$ will then show small correction terms, if the initial guess is close to being correct.

\section{Appendix}

In this development we wish to convert the expression

$$
\begin{aligned}
C(\mathbf{v} ; t)= & \exp \left[-N \bar{v} \pi B^{2} t+i \mathbf{k} \cdot \mathbf{v} t\right] \\
& +N \int_{0}^{t} d t^{\prime} \exp \left[-N \bar{v} \pi B^{2} t^{\prime}+i \mathbf{k} \cdot \mathbf{v} t^{\prime}\right] \\
& \times \int^{B} \int d \mathbf{b} d \mathbf{u} f(\mathbf{u})|\mathbf{v}-\mathbf{u}| S(\mathbf{v} ; \mathbf{b}, \mathbf{u}) C\left(\mathbf{v}^{\prime} ;\left(t-t^{\prime}\right)\right)
\end{aligned}
$$

which was given as (9) into the form as shown in (10) of the text. We begin by defining a reduced correlation function:

$$
D(\mathbf{v} ; t)=C(\mathbf{v} ; t) \exp \left[N \bar{v} \pi B^{2} t-i \mathbf{k} \cdot \mathbf{v} t\right]
$$

which satisfies

$$
\begin{aligned}
D(\mathbf{v} ; t)= & 1+N \int_{0}^{t} d t^{\prime} \int^{B} \int d \mathbf{b} d \mathbf{u} f(\mathbf{u})|\mathbf{v}-\mathbf{u}| S(\mathbf{v} ; \mathbf{b}, \mathbf{u}) \\
& \times \exp \left[-i \mathbf{k} \cdot\left(\mathbf{v}-\mathbf{v}^{\prime}\right)\left(t-t^{\prime}\right)\right] D\left(\mathbf{v}^{\prime} ;\left(t-t^{\prime}\right)\right)
\end{aligned}
$$

It now simplifies things if one replaces $\left(t-t^{\prime}\right)$ by $t^{\prime}$ itself, for mathematical convenience, giving simply

$$
\exp \left[-i \mathbf{k} \cdot\left(\mathbf{v}-\mathbf{v}^{\prime}\right) t^{\prime}\right] D\left(\mathbf{v}^{\prime} ; t^{\prime}\right)
$$

as the latter terms in (A.3). Now, differentiate $D(v ; t)$ with respect to $t$, utilizing (A.3),

$$
\begin{aligned}
\frac{d D(\mathbf{v} ; t)}{d t}= & N \int^{B} \int d \mathbf{b} d \mathbf{u} f(\mathbf{u})|\mathbf{v}-\mathbf{u}| S(\mathbf{v} ; \mathbf{b}, \mathbf{u}) \\
& \times \exp \left[-i \mathbf{k} \cdot\left(\mathbf{v}-\mathbf{v}^{\prime}\right) t\right] D\left(\mathbf{v}^{\prime} ; t\right) .
\end{aligned}
$$

Divide by $D(\mathbf{v} ; t)$ itself, to obtain the logarithmic derivative, and noting the initial condition $D(\mathbf{v} ; 0)=1$, this can be integrated and exponentiated to the form

$$
\begin{aligned}
D(\mathbf{v} ; t)=\exp \left[N \int_{0}^{t} d t^{\prime} \int^{B} \int d \mathbf{b} d \mathbf{u} f(\mathbf{u})|\mathbf{v}-\mathbf{u}|\right. \\
\left.\quad \times S(\mathbf{v} ; \mathbf{b}, \mathbf{u})\left(\frac{D\left(\mathbf{v}^{\prime} ; t^{\prime}\right)}{D\left(\mathbf{v} ; t^{\prime}\right)}\right) \exp \left[-i \mathbf{k} \cdot\left(\mathbf{v}-\mathbf{v}^{\prime}\right) t^{\prime}\right]\right] .
\end{aligned}
$$

Changing back to the corresponding equation in $C(v ; t)$, we have

$$
\begin{aligned}
C(\mathbf{v} ; t)=\exp [ & i \mathbf{k} \cdot \mathbf{v} t-N \bar{v} \pi B^{2} t+N \int_{0}^{t} d t^{\prime} \int^{B} \int d \mathbf{b} d \mathbf{u} \\
& \left.\times f(\mathbf{u})|\mathbf{v}-\mathbf{u}| S(\mathbf{v} ; \mathbf{b}, \mathbf{u})\left(\frac{C\left(\mathbf{v}^{\prime} ; t^{\prime}\right)}{C\left(\mathbf{v} ; t^{\prime}\right)}\right)\right] .
\end{aligned}
$$

It now looks as if the troublesome exponential $\exp \left[-N \bar{v} \pi B^{2} t\right]$ still remains, but with $\bar{v}$ being the average relative speed of collision, we note that

$$
\bar{v} \pi B^{2} t=\int_{0}^{t} d t^{\prime} \int_{0}^{B} \int d \mathbf{b} d \mathbf{u} f(\mathbf{u})|\mathbf{v}-\mathbf{u}|
$$


itself, so that the final result

$$
\begin{aligned}
C(\mathbf{v} ; t)=\exp [ & i \mathbf{k} \cdot \mathbf{v} t-N \int_{0}^{t} d t^{\prime} \iint d \mathbf{b} d \mathbf{u} f(\mathbf{u})|\mathbf{v}-\mathbf{u}| \\
& \left.\times\left(1-S(\mathbf{v} ; \mathbf{b}, \mathbf{u})\left(\frac{C\left(\mathbf{v}^{\prime} ; t^{\prime}\right)}{C\left(\mathbf{v} ; t^{\prime}\right)}\right)\right)\right]
\end{aligned}
$$

is achieved, which is shown as (10) of the text. Importantly, the actual specification of $B$ is no longer necessary with integration on all physically relevant $\mathbf{b}$ values being implied.

\section{Acknowledgments}

The author takes pleasure in acknowledging helpful conversations with R. Ciuryło, J. C. Lewis, J. P. Looney, and R. H. Tipping, and for help in manuscript preparation by $\mathrm{A}$. Grugan.

\section{References}

[1] P. R. Berman, "Speed-dependent collisional width and shift parameters in spectral profiles," Journal of Quantitative Spectroscopy and Radiative Transfer, vol. 12, no. 9, pp. 1331-1342, 1972.

[2] J. Ward, J. Cooper, and E. W. Smith, "Correlation effects in the theory of combined doppler and pressure broadeningI. Classical theory," Journal of Quantitative Spectroscopy and Radiative Transfer, vol. 14, no. 7, pp. 555-590, 1974.

[3] I. Shannon, M. Harris, D. R. McHugh, and E. L. Lewis, "Low-pressure spectral line profiles: an analysis in terms of symmetric speed-dependent Voigt profiles," Journal of Physics $B$, vol. 19, no. 10, pp. 1409-1424, 1986.

[4] R. H. Dicke, "The effect of collisions upon the doppler width of spectral lines," Physical Review, vol. 89, no. 2, pp. 472-473, 1953.

[5] J.-M. Hartmann, C. Boulet, and D. Robert, Collisional Effects on Molecular Spectra, Elsevier, New York, NY, USA, 2008.

[6] M. Baranger, "Problem of overlapping lines in the theory of pressure broadening," Physical Review, vol. 111, no. 2, pp. 494504, 1958.

[7] A. C. Kolb and H. Griem, "Theory of line broadening in multiplet spectra," Physical Review, vol. 111, no. 2, pp. 514$521,1958$.

[8] K. Shimoda, Ed., High-Resolution Laser Spectroscopy, Springer, Heidelberg, Germany, 1976.

[9] R. M. Herman and E. W. Weber, "Comprehensive theory for line broadening in high-resolution and nonlinear spectroscopies: application to the Na D lines," Journal of Physics B, vol. 16, no. 8, pp. 1323-1341, 1983.

[10] L. Galatry, "Simultaneous effect of doppler and foreign gas broadening on spectral lines," Physical Review, vol. 122, no. 4, pp. 1218-1223, 1961.

[11] P. W. Anderson and J. D. Talman, Pressure Broadening of Spectral Lines at General Pressures, Bell Telephone System Technical Publications, Monograph 3117, 1956.

[12] G. Traving, Über die Theorie der Druckverbreiterung von Spectrallinien, G. Braun, Karlsruhe, Germany, 1960.

[13] J. Szudy and W. E. Baylis, "Unified Franck-Condon treatment of pressure broadening of spectral lines," Journal of Quantitative Spectroscopy and Radiative Transfer, vol. 15, no. 7-8, pp. 641-668, 1975.
[14] J. Szudy and W. E. Baylis, "Asymmetry in pressure-broadened spectral lines," Journal of Quantitative Spectroscopy and Radiative Transfer, vol. 17, pp. 681-684, 1977.

[15] A. S. Pine, "Collisional narrowing of HF fundamental band spectral lines by Neon and Argon," Journal of Molecular Spectroscopy, vol. 82, pp. 435-448, 1980.

[16] A. S. Pine and J. P. Looney, " $\mathrm{N}_{2}$ and air broadening in the fundamental bands of $\mathrm{HF}$ and $\mathrm{HCl}$," Journal of Molecular Spectroscopy, vol. 122, no. 1, pp. 41-55, 1987.

[17] A. S. Pine and R. Ciurylo, "Multispectrum fits of Arbroadened HF with a generalized asymmetric lineshape: effects of correlation, hardness, speed dependence, and collision duration," Journal of Molecular Spectroscopy, vol. 208, no. 2, pp. 180-187, 2001.

[18] R. Wehr, A. Vitcu, R. Ciuryło, F. Thibault, J. R. Drummond, and A. D. May, "Spectral line shape of the $P(2)$ transition in CO-Ar: uncorrelated ab initio calculation," Physical Review A, vol. 66, no. 6, Article ID 062502, 7 pages, 2002.

[19] R. Wehr, R. Ciuryło, A. Vitcu, F. Thibault, J. R. Drummond, and A. D. May, "Dicke-narrowed spectral line shapes of CO in Ar: experimental results and a revised interpretation," Journal of Molecular Spectroscopy, vol. 235, no. 1, pp. 54-68, 2006.

[20] J.-M. Colmont, B. Bakri, F. Rohart, et al., "Intercomparison between ozone-broadening parameters retrieved from millimetre-wave measurements by using different techniques," Journal of Molecular Spectroscopy, vol. 231, no. 2, pp. 171-187, 2005.

[21] F. Rohart, G. Włodarczak, J.-M. Colmont, G. Cazzoli, L. Dore, and C. Puzzarini, "Galatry versus speed-dependent Voigt profiles for millimeter lines of $\mathrm{O}_{3}$ in collision with $\mathrm{N}_{2}$ and $\mathrm{O}_{2}$," Journal of Molecular Spectroscopy, vol. 251, no. 1-2, pp. 282292, 2008.

[22] D. Lisak, J. T. Hodges, and R. Ciuryło, "Comparison of semiclassical line-shape models to rovibrational $\mathrm{H}_{2} \mathrm{O}$ spectra measured by frequency-stabilized cavity ring-down spectroscopy," Physical Review A, vol. 73, no. 1, Article ID 012507, 13 pages, 2006.

[23] D. Priem, F. Rohart, J.-M. Colmont, G. Włodarczak, and J.-P. Bouanich, "Lineshape study of the $J=3 \leftarrow 2$ rotational transition of CO perturbed by $\mathrm{N}_{2}$ and $\mathrm{O}_{2}$," Journal of Molecular Structure, vol. 517-518, pp. 435-454, 2000.

[24] R. Wehr, A. Vitcu, F. Thibault, J. R. Drummond, and A. D. May, "Collisional line shifting and broadening in the fundamental P-branch of CO in Ar between 214 and $324 \mathrm{~K}$," Journal of Molecular Spectroscopy, vol. 235, no. 1, pp. 69-76, 2006.

[25] R. Wehr, A. Vitcu, F. Thibault, J. R. Drummond, and A. D. May, "Erratum to "Collisional line shifting and broadening in the fundamental P-branch of CO in Ar between 214 and 324 K" [J. Mol. Spectrosc. 235 (2006) 69-76]," Journal of Molecular Spectroscopy, vol. 237, no. 1, p. 126, 2006.

[26] R. L. Farrow, L. A. Rahn, G. O. Sitz, and G. J. Rosasco, "Observation of a speed-dependent collisional inhomogeneity in $\mathrm{H}_{2}$ vibrational line profiles," Physical Review Letters, vol. 63, no. 7, pp. 746-749, 1989.

[27] F. Chaussard, R. Saint-Loup, H. Berger, et al., "Speeddependent line profile: a test of a unified model from the Doppler to the collisional regime for molecule-molecule collisions," Journal of Chemical Physics, vol. 113, no. 12, pp. 4951-4956, 2000.

[28] S. G. Rautian and I. I. Sobel'man, "The effect of collisions on the Doppler broadening of spectral lines," Soviet Physics Uspekhi, vol. 9, pp. 701-716, 1967. 
[29] R. M. Herman, "Toward a unified impact theory for everything concerning neutral species line shapes," in CP559, Spectral Line Shapes: Volume 11, 15th ICSLS, J. Seidel, Ed., pp. 237-243, American Institute of Physics, 2001.

[30] J. P. Looney, Comprehensive theory for the broadening, shifting and narrowing of $\mathrm{HF}$ and $\mathrm{HCl}$ fundamental band absorption profiles, Ph.D. thesis, The Pennsylvania State University, 1987.

[31] Priem, et al., loc. cit.

[32] S. Green and J. Hutson, "Spectral line shape parameters for $\mathrm{HF}$ in a bath of Ar are accurately predicted by a potential inferred from spectra of the van der Waals dimer," The Journal of Chemical Physics, vol. 100, no. 2, pp. 891-898, 1994.

[33] P. W. Anderson, "Pressure broadening in the microwave and infra-red regions," Physical Review, vol. 76, no. 5, pp. 647-661, 1949.

[34] C. J. Tsao and B. Curnutte, "Line-widths of pressurebroadened spectral lines," Journal of Quantitative Spectroscopy and Radiative Transfer, vol. 2, no. 1, pp. 41-91, 1962.

[35] J. Jarecki and R. M. Herman, "Widths and shifts of HF vibration-rotation absorption lines induced by pressure of rare gases," Journal of Quantitative Spectroscopy and Radiative Transfer, vol. 15, no. 9, pp. 707-726, 1975.

[36] J. S. Murphy and J. E. Boggs, "Collision broadening of rotational absorption lines. I. Theoretical formulation," The Journal of Chemical Physics, vol. 47, no. 2, pp. 691-702, 1967.

[37] R. M. Herman, "Impact theory of the noble-gas-broadened $\mathrm{HCl}$ vibration-rotation lines," Journal of Quantitative Spectroscopy and Radiative Transfer, vol. 3, p. 443, 1963.

[38] R. H. Tipping and R. M. Herman, "Impact theory for the noble gas pressure-induced $\mathrm{HCl}$ vibration-rotation and pure rotation line widths-I," Journal of Quantitative Spectroscopy and Radiative Transfer, vol. 10, no. 8, pp. 881-896, 1970.

[39] R. M. Herman and R. H. Tipping, "Impact theory for the noble gas pressure-induced $\mathrm{HCl}$ vibration-rotation and pure rotation line shifts-II," Journal of Quantitative Spectroscopy and Radiative Transfer, vol. 10, no. 8, pp. 897-908, 1970. 


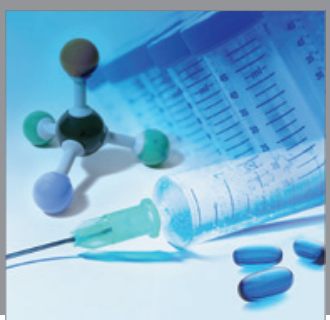

International Journal of

Medicinal Chemistry

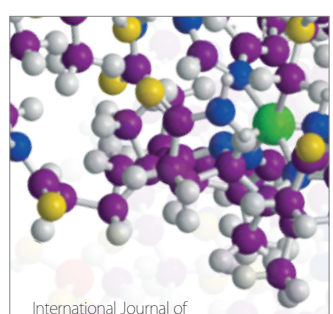

Carbohydrate Chemistry

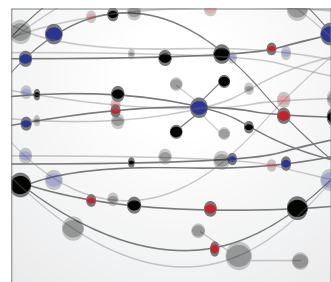

The Scientific World Journal
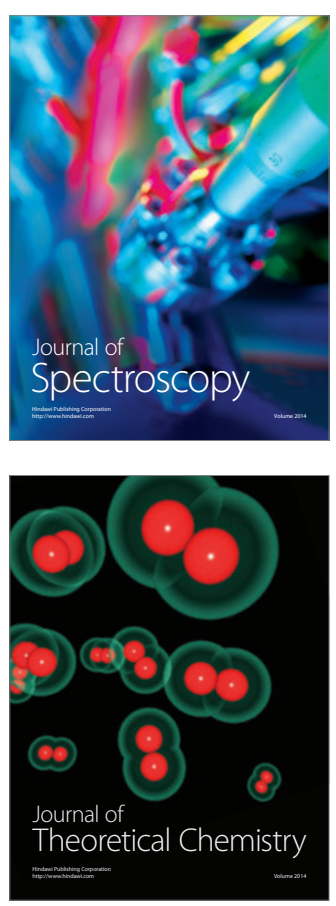
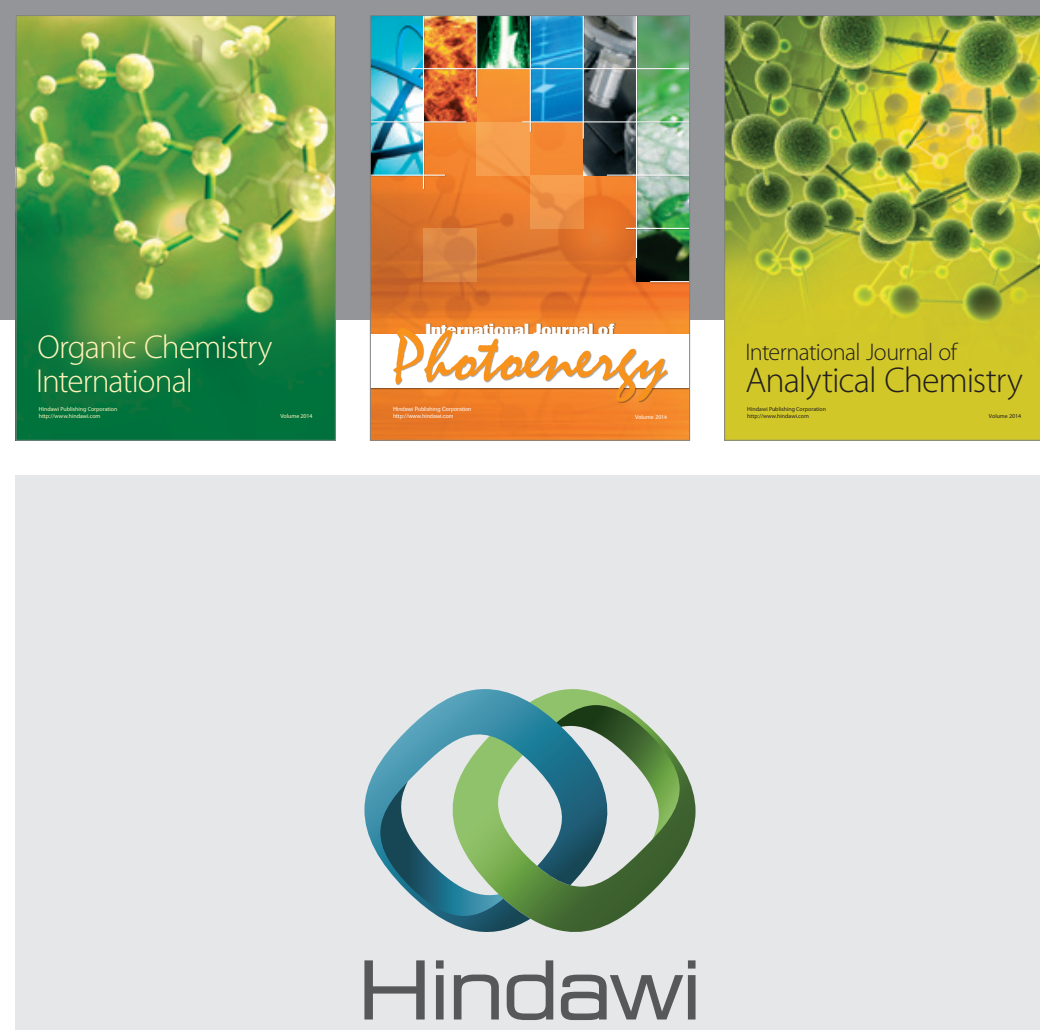

Submit your manuscripts at

http://www.hindawi.com
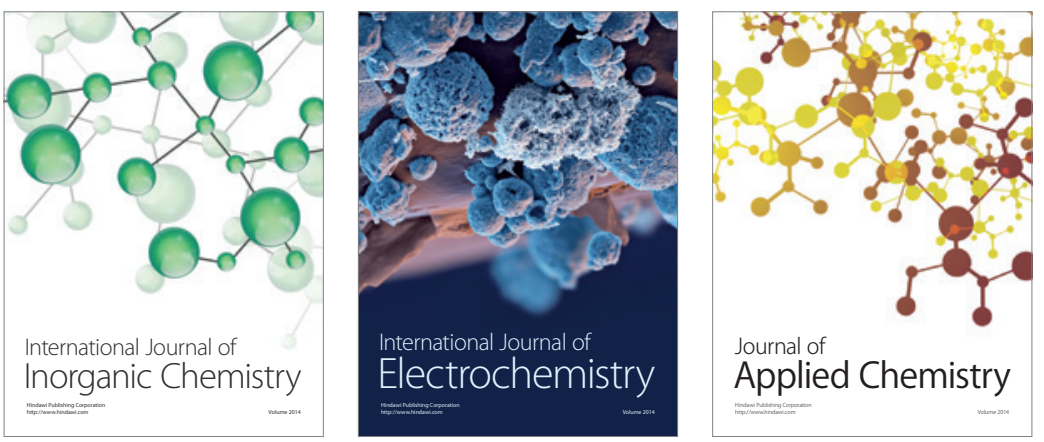

Journal of

Applied Chemistry
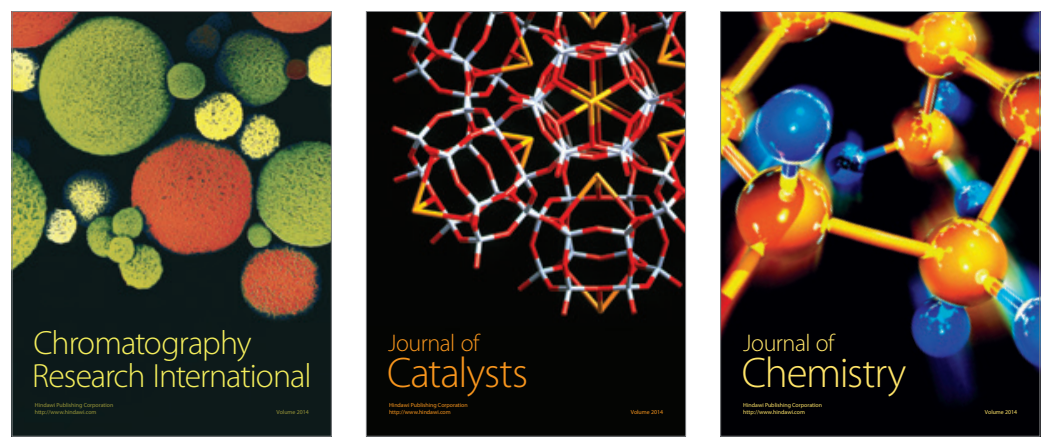
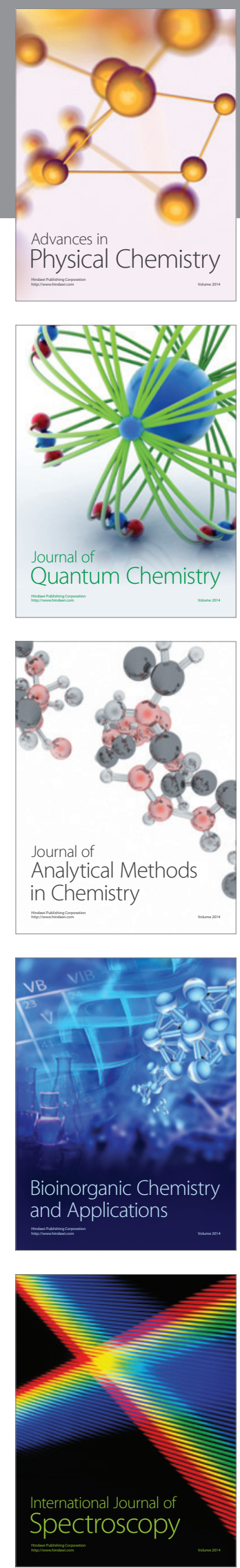